\title{
Sources of graft restriction after single lung transplantation for emphysema
}

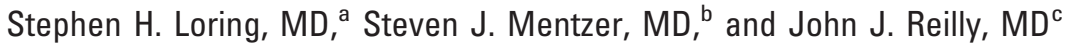

From the Department of Anesthesia and Critical Care, ${ }^{\mathrm{a}}$ Beth Israel Deaconess Medical Center and Harvard Medical School, the Division of Thoracic Surgery, ${ }^{\mathrm{b}}$ Brigham and Women's Hospital and Harvard Medical School, and the Division of Pulmonary and Critical Care Medicine, ${ }^{\mathrm{c}}$ Brigham and Women's Hospital and Harvard Medical School, Boston, Mass.

This work was supported by grant HL52586 from the National Institutes of Health.

Received for publication Oct 5, 2006; revisions received Jan 3, 2007; accepted for publication Feb 12, 2007.

Address for reprints: Stephen H. Loring, MD, 330 Brookline Ave, Dana 717, Boston MA 02215 (E-mail: sloring@bidmc.harvard. edu).

J Thorac Cardiovasc Surg 2007;134:204-9

$0022-5223 / \$ 32.00$

Copyright $\odot 2007$ by The American Association for Thoracic Surgery

doi:10.1016/j.jtcvs.2007.02.008
Objective: After single lung transplantation for emphysema, the volume of the graft at total lung capacity is usually less than its predicted volume in the donor. We sought to determine the contributions of donor-recipient size matching, postoperative native lung hyperinflation, and postoperative chest wall volume reduction to graft restriction after transplantation.

Methods: In 19 patients, we estimated individual lung volumes from thoracic computed tomographs taken near total lung capacity before and after single lung transplantation for emphysema to analyze sources of graft restriction. Pulmonary function was assessed by spirometry, and in 5 patients, inspiratory function was assessed with esophageal manometry.

Results: Graft volumes after transplantation were $54 \% \pm 17 \%$ of those predicted for the donors (mean $\pm \mathrm{SD}, P<.0001$ ), and pulmonary function after transplantation was significantly correlated with graft volume. The greatest contribution to graft restriction was the decrease in chest wall volume after transplantation, which was $-0.87 \mathrm{~L}(-31 \% \pm 29 \%$ of the graft's predicted volume; $P<.0001)$. Volume expansion of the contralateral lung contributed $-0.44 \mathrm{~L}(-18 \% \pm 24 \% ; P=$ .0018). Other effects, including donor-patient size matching, were not significant. In 5 patients, the maximum negative inspiratory esophageal pressure at total lung capacity was low $\left(-6 \pm 2 \mathrm{~cm} \mathrm{H}_{2} \mathrm{O}\right.$, normal range $\sim-17$ to $\left.-29 \mathrm{~cm} \mathrm{H}_{2} \mathrm{O}\right)$.

Conclusions: After single lung transplantation for emphysema, decreased volume of the chest wall was more important than increased volume of the native lung in causing restriction of the graft and decreased pulmonary function. Chest wall restriction is likely due to diminished inspiratory muscle function.

$\mathrm{S}$ ingle lung transplantation (SLT) has become an accepted therapy for endstage emphysema. Early concerns that native lung hyperinflation would restrict expansion of the graft ${ }^{1,2}$ were allayed by subsequent studies showing good ventilatory function after SLT, despite persistent hyperinflation of the native lung. ${ }^{3-7}$ Nonetheless, there is evidence that after SLT for emphysema the graft is restricted by encroachment of the native lung. ${ }^{8,9}$ Whether such restriction of the graft results in reduced ventilatory function is less certain. Brunsting and associates ${ }^{8}$ found that after SLT, ventilatory function of the graft (inferred from a ventilation scan) was nearly normal despite a lower than normal volume at TLC. Estenne and coworkers $^{10}$ found that graft volumes were less than normal at TLC, but at functional residual capacity (FRC) they were not different from those of control subjects.

After SLT for emphysema, graft expansion depends not only on the properties of the graft and contralateral native lung but also on the chest wall. Thus, there are two major causes of extrinsic restriction of the graft: hyperinflation of the native lung and chest wall volume reduction. Using planimetry of chest radiographs in 7 patients after SLT, Cheriyan and colleagues ${ }^{9}$ showed that graft 


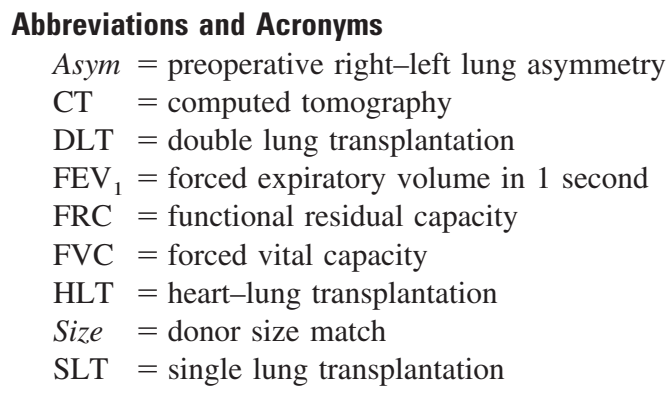

volumes were persistently low at about $33 \%$ of the predicted TLC, and there was an associated progressive increase in volume of the native lung. However, the chest wall volume remained slightly greater than normal in these subjects. Similarly, Brunsting and associates ${ }^{8}$ reported normal values of TLC in 9 patients after SLT. They also showed that postoperative ventilatory function of the graft was correlated with preoperative chest wall volume and concluded that graft function was largely dependent on postoperative chest wall (ie, total lung) volume. ${ }^{8}$ Transient postoperative chest wall restriction has been reported after double lung transplantation (DLT) and heart-lung transplantation (HLT), but this effect resolves after several months, and overall lung function is correlated with the postoperative total chest wall volume or TLC.

We wondered to what extent graft inflation at TLC in patients after SLT might be limited by these two mechanisms: expansion of the native lung after transplantation and postoperative reduction in the volume of the chest wall. Native lung hyperinflation can be addressed with volume reduction surgery on the native lung, whereas significant chest wall restriction after transplantation would invite investigation of its causes and strategies for its prevention. To assess the relative importance of each mechanism of graft underinflation, we retrospectively examined the changes in the volumes of the chest wall, graft, and contralateral lungs in subjects before and after SLT for emphysema.

\section{Patients and Methods}

Subjects were 19 of approximately 54 patients evaluated physiologically before SLT for emphysema at Brigham and Women's Hospital between November 1990 and September 2003. All subjects had smoked, 2 had $\alpha_{1}$-antitrypsin deficiency, and 3 had undergone prior lung volume reduction surgery. SLTs (9 right, 10 left) were performed via posterolateral thoracotomy without bypass, with donor-recipient size matching according to height. Subjects were included in this analysis if they had thoracic computed tomographic (CT) scans taken both before and after SLT at a time when there was no infection, rejection (including bronchiolitis obliterans syndrome), or other condi- tion that could have caused restriction of the graft. The CT scans were obtained during a breath hold after a deep (near maximal) inhalation, as is standard practice. In subjects in whom multiple postoperative CTs were available, the CT scan showing maximal graft volume, hereafter called the "best" postoperative CT, was chosen for analysis. Best CTs were taken from 23 days to 37 months after transplantation (425 \pm 358 days, mean $\pm \mathrm{SD}$ ). All subjects gave informed written consent for the protocol, which was approved by the Partners Human Research Committee.

To determine the air volume contained in each lung, the left and right lungs were highlighted on each slice to the border of the mediastinum, without including main-stem bronchial or tracheal air volumes, and the area was multiplied by slice thickness to obtain volume. The density of each volume was evaluated by interpolation between blood density (tissue density) and air density to calculate the volume of air contained. Air volumes in the slices were summed to obtain the air volume contained in left and right lungs. Total lung volume was taken as the air volume contained in both lungs.

In 17 of these 19 patients, ventilatory function after transplantation was assessed by spirometry, including forced vital capacity (FVC) and forced expiratory volume in 1 second $\left(\mathrm{FEV}_{1}\right)$, measured within 4 months of the best CT. CT scans and FVCs were obtained without prior bronchodilation, but we did not require patients to omit their routine bronchodilator treatments.

A subset of 5 of these patients had respiratory mechanical studies within 57 days of the best CT. The maximal negative inspiratory pleural pressure exerted by the chest wall at TLC, which is the negative of the elastic recoil pressure of the lungs at TLC, was measured with an esophageal balloon catheter by techniques described previously ${ }^{11}$ to infer the contribution of inspiratory muscle weakness to low total volume after transplantation.

\section{Analysis}

To evaluate the relative importance of postoperative chest wall volume reduction and postoperative native lung expansion to postoperative restriction of graft volume at TLC, we apportioned the volume change of the transplanted lung, beginning with its predicted volume in the donor to its volume in the recipient after transplantation, among 5 separate effects: (1) donor size match, (2) preoperative hyperinflation, (3) preoperative left-right lung asymmetry, (4) postoperative chest wall volume reduction, and (5) native lung hyperexpansion. In the equations that follow, $P t$ and Donor indicate the patient and donor, PredTLC indicates a predicted TLC (based on height, age, sex, and race when available), Pre and Post indicate preoperative and postoperative volumes, Vtot indicates the sum of left and right lung air volumes measured on CT scans, and Vipsi indicates preoperative air volume on the side ipsilateral to the transplant (ie, the volume of the excised lung). Fx is the expected "standard" volume fraction of the transplanted lung relative to 


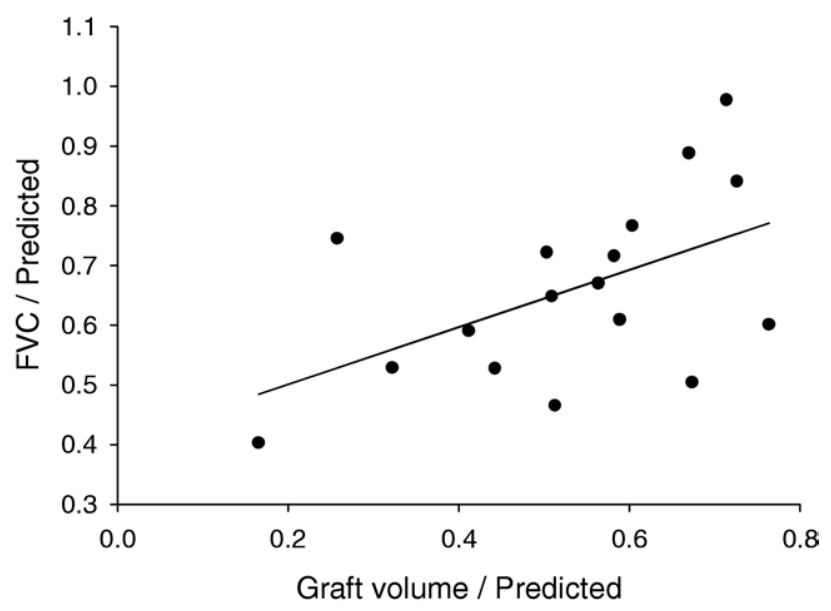

Figure 1. FVC and graft volume in patients after SLT. Graft volume at TLC was significantly correlated with ventilatory function $(P=$ .0343).

total lung volume, which we took to be 0.53 for right and 0.47 for left lung transplantation.

Changes in the size of the graft, and therefore the graft volume, can be attributed conceptually to the following extrinsic effects:

1. Donor size match (Size), the change in the graft volume resulting from the difference between patient and donor body sizes, is the difference between the predicted volumes of the graft in the donor and ipsilateral lung in the patient, assuming a standard volume fraction.

$$
\text { Size } \equiv \text { Fx } \cdot(\text { PtPredTLC }- \text { DonorPredTLC })
$$

A lung from a large donor will tend to be less expanded in a small recipient.

2. Preoperative hyperinflation (Hyperinf), the change in the graft volume resulting from chest wall hyperinflation before transplantation, is the difference between the patient's actual total volume and predicted TLC.

$$
\text { Hyperinf } \equiv F x \cdot(\text { PtPreVtot }- \text { PtPredTLC })
$$

The graft volume would tend to increase owing to the enlargement of the chest wall containing the patient's ipsilateral lung, assuming standard volume fraction.

3. Preoperative left-right lung asymmetry (Asym) is the change in the graft volume owing to abnormally unequal left and right lung volumes before operation.

$$
\text { Asym } \equiv \text { PtPreVipsi }- \text { Fx } \cdot(\text { PtPreVtot })
$$

Although the left lung is normally slightly smaller than the right, which does not affect graft inflation, an abnormally asymmetrical distribution of lung volume before transplantation could affect expansion of the graft. For example, a right lung graft would tend to be less inflated if it replaced an abnormally small right lung.

4. Postoperative chest wall volume change (Chest), the change in the graft volume resulting from changed overall chest wall volume after transplantation, is the difference between total intrathoracic gas volumes before and after transplantation.

$$
\text { Chest } \equiv(\text { PtPostVtot }- \text { PtPreVtot })
$$

5. Native lung hyperexpansion (Native), the change in the graft volume owing to postoperative changes in the contralateral (native) lung volume, is the change in the contralateral lung volume (total lung volume minus ipsilateral lung volume) from before to after transplantation.

$$
\begin{aligned}
\text { Native } \equiv(\text { PtPreVtot }- \text { PtPreVipsi }) & -(\text { PtPostVtot } \\
& - \text { PtPostVipsi })
\end{aligned}
$$

The sum of these effects, algebraically and conceptually, is the difference between the postoperative volume of the graft at TLC (PtPostVipsi) and the predicted volume of that lung in the donor ( $F x$. DonorPredTLC). The effects are reported as a fraction of the predicted volume of the graft in the donor. Note that the effects resulting from Hyperinf and Asym are concurrent, as are the effects resulting from Chest and Native. The order of calculation and presentation of effects in each pair is arbitrary and could be reversed without loss of generality.

Statistical significance of the mean effects was assessed by paired $t$ tests and Wicoxon signed rank tests, and correlations were tested by linear regression.

\section{Results}

Overall, graft volume after transplantation averaged 54\% \pm $17 \%$ of its predicted volume in the donor, a change of $-1.26 \pm 0.49 \mathrm{~L}(P<.0001)$.

Pulmonary function after transplantation was better in patients with greater graft volume. The FVC and the FEV relative to predicted were significantly correlated with the relative volume of the graft (FVC: $R^{2}=0.27, P=.0343$, Figure $1 ; \mathrm{FEV}_{1}: R^{2}=0.24, P=.0486$ ). By contrast, native lung size had a weak negative effect on lung function that did not reach significance.

Contributions to graft underinflation of Size, Hyperinf, Asym, Chest, and Native for individual subjects are depicted in Figure 2, and these effects are shown as sequential cumulative contributions in Figure 3. The largest effect contributing to the decrease in the graft volume was the change in chest wall volume after transplantation (Chest), which was $-0.87 \mathrm{~L}$ or $-31 \% \pm 29 \%$ of the graft's predicted volume $(P<.0001)$. Chest wall volumes decreased 


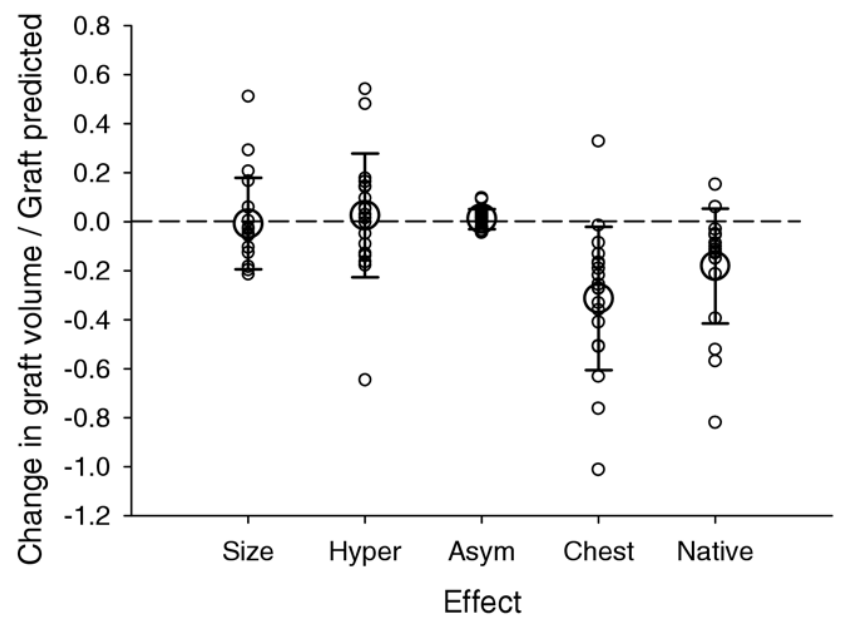

Figure 2. Relative changes in graft volume after SLT attributable to donor-patient size differences (Size), preoperative hyperinflation (Hyper), preoperative volume exchange between lungs (Asym), postoperative change in overall chest wall size (Chest), and postoperative increase in native lung volume (Native). Effects for each patient are shown with mean and SD. The largest effect was Chest $(P<.0001)$ followed by Native $(P=.0018)$; other effects were not significant for the group. (Note: individual effects can appear to decrease graft volume by more than $100 \%$ because of other compensating effects.)

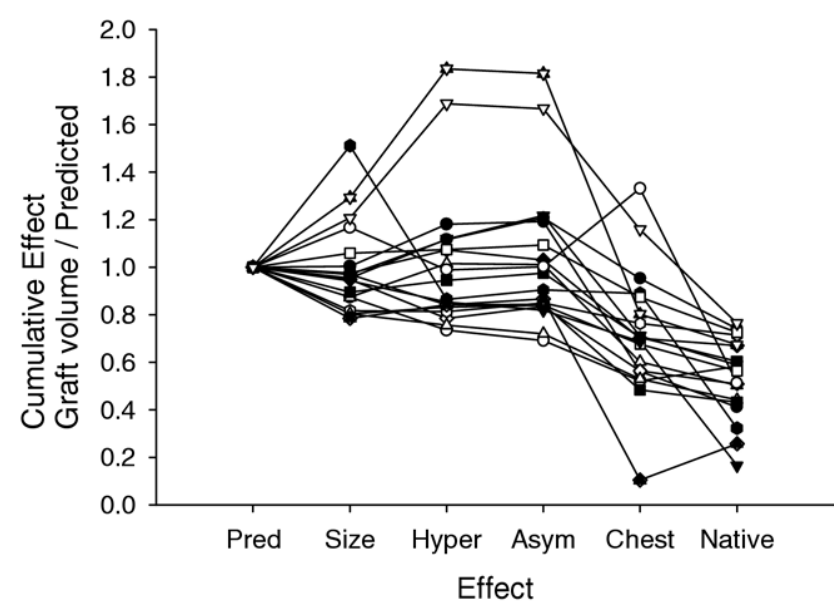

Figure 3. Cumulative effects of Size, Hyper, Asym, Chest, and Native on the change in graft volume in individual subjects. The effects combined to reduce graft volume below predicted in all subjects. For explanation of terms, see Figure 2.

after transplantation in all but one subject, and total air volumes measured on CT scans after transplantation were less than predicted TLC in 16 of 19 subjects, on average $87 \% \pm 15 \%$ of predicted TLC $(P=.0006$, Figure 4$)$. Volume expansion of the contralateral lung after transplan- tation (Native) was also significant, accounting for an average loss of graft volume of $-0.44 \mathrm{~L}$ or $-18 \% \pm 24 \%$ of the graft's predicted volume $(P=.0018)$. The other effects, Hyperinf, Size, and Asym, had smaller, inconsistent effects that were not statistically significant.

The relative size of the donor lung was not significantly correlated with the FVC (percent predicted), $\mathrm{FEV}_{1}$ (percent predicted), or the size of the graft as a fraction of the predicted size in the donor.

Five of the patients had chest wall mechanics measured after transplantation within 2 months of the best postoperative CT. Negative inspiratory esophageal pressure at TLC was $-6 \pm 2 \mathrm{~cm} \mathrm{H}_{2} \mathrm{O}$ (range -5 to $-8 \mathrm{~cm} \mathrm{H}_{2} \mathrm{O}$ ), substantially less negative than the normal range ${ }^{12}(\sim-17$ to -29 $\mathrm{cm} \mathrm{H}_{2} \mathrm{O}$ ), suggesting that failure to expand the chest was not due to low pulmonary compliance but to factors intrinsic to the chest wall, such as inspiratory muscular weakness.

\section{Discussion}

In our subjects, transplanted lungs were smaller than predicted at TLC, as has been observed in numerous other studies of SLT for emphysema (eg, studies by Cheriyan, ${ }^{9}$ Estenne, ${ }^{10}$ and their associates). Surprisingly, we found that reduction of the chest wall volume was quantitatively more important than postoperative expansion of the native lung in limiting the size of graft. Both of these causes of graft restriction have been described in the literature, but we are not aware of previous reports of the relative magnitudes of Chest and Native effects. The impression one gets from thoracic CT scans is that the native lung occupies most of the thoracic volume, crowding the graft and limiting its expansion. It is tempting to attribute this appearance to expansion of the native lung at the time of transplantation or

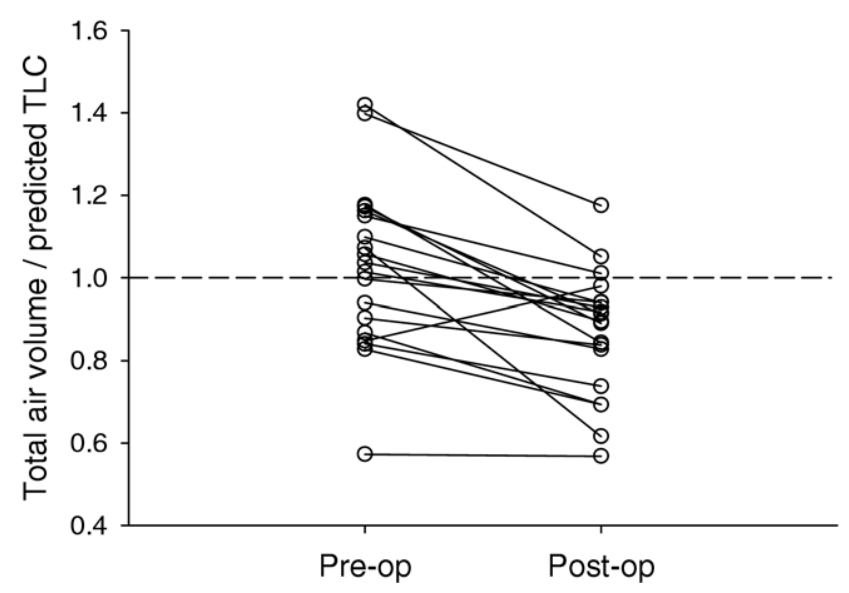

Figure 4. Overall chest wall volume, as assessed by air volume measured on CT scans, before and after SLT for emphysema. Air volume decreased in all subjects but one $(P<.0006)$. TLC, Total lung capacity. 
to its progressive expansion after transplantation, whereas our results suggest that the reduction in chest wall volume at transplantation, coupled with relative preservation of the volume of the native lung, accounts for most of the volume decrease seen in the graft.

Our findings should not be taken to imply that if the chest wall were not restricted in volume after transplantation, the graft would be normally inflated. It is more likely that greater chest wall expansion after transplantation would preferentially expand the native lung, leaving the graft still somewhat underinflated. Indeed, expansion of the contralateral lung (Native) contributed an average of 18\% (of graft predicted) to the reduction in graft volume.

In this study, total lung volumes were estimated from thoracic CT scans taken after deep inhalation in the supine position. Because CT scans were not taken at regular intervals, the best CT may have missed the time when graft volume was at its maximum. The deep inhalation before the CT scan may not have been truly maximal, and 50 to 100 $\mathrm{mL}$ of gas in the extrapulmonary airways was not measured by our method. Furthermore, TLC is reportedly slightly less in the supine position than upright. For these reasons, it could be argued that total volumes measured by CT would be systematically less than TLC measured by helium dilution or plethysmography, and therefore that our finding that postoperative total lung volume was less than predicted TLC could be due in part to differences between methods of measurement. However, the differences between TLC measured by these methods are likely to be small. In over 80 subjects studied at our hospitals, TLC values measured by helium dilution were not systematically different from those measured by $\mathrm{CT}$ scan. Moreover, the major findings and conclusions of our study, which were based on comparisons among volumes obtained with a single technique, should not be affected by the method of measurement.

Although chest wall restriction has been recognized as a major factor producing a restrictive ventilatory defect in the first few months after DLT and HLT, ${ }^{12,13}$ TLC usually returns to preoperative or predicted values within a year. Glanville and colleagues ${ }^{14}$ showed that the restrictive defect in 12 patients after HLT was due not to low compliance of the transplanted lungs, which had normal pressure-volume curves, but to reduced inspiratory muscle force, as we found in a subset of our subjects. In that study, the postoperative TLC (percent predicted) was highly correlated with maximal inspiratory pressure. Other studies have reported that TLC remained in a normal range after transplantation. For example, in 33 patients after HLT or DLT for hyperinflated lung disease, Pinet and Estenne ${ }^{15}$ showed that TLC decreased after transplantation to normal predicted values and remained stable for up to 3 years, but that FRC remained elevated, suggesting that the chest wall had retained structural adaptations to longstanding lung hyperinflation. Sim- ilarly, Guignon and colleagues ${ }^{16}$ found normal TLC values after HLT, but patients who had HLT for cystic fibrosis had persistent hyperinflation at FRC and residual volume, which they attributed to persistent adaptations of the growing chest wall to chronically hyperinflated lungs. Thus, our results differ from most reports of restriction after DLT or HLT, in which the restrictive defect resolved with time.

Several studies have shown that postoperative lung function does not depend on an exact match of donor and patient lungs sizes, both in $\mathrm{DLT}^{13}$ and in SLT. ${ }^{8}$ There is likewise no evidence in our subjects that smaller donor lung volumes were less likely to be restricted or to have better postoperative ventilatory function.

The degree to which chest wall volume reduction causes unequal restriction of graft and native lungs depends on the degree to which the chest wall resists asymmetrical expansion of the left and right lungs. ${ }^{17}$ For example, if the mediastinum could not be displaced laterally by the difference in pleural pressures surrounding native and transplanted lungs, and if the thorax could expand only symmetrically, chest wall volume reduction would cause symmetrical reductions in graft and native lung volumes, resulting in better ventilatory function and less restriction of the graft. Mediastinal displacement and asymmetrical expansion of the two hemithoraces exacerbates the effects of chest wall restriction in the setting of unilateral emphysema. ${ }^{18,19}$ On the other hand, a recent experimental study suggests that the unequal displacement of the diaphragm after SLT may facilitate diaphragmatic function on the transplanted side, improving ventilation of the graft $^{20}$ despite restriction of graft volume at TLC.

The etiology of postoperative chest wall volume reduction is not clear. Postoperative chest wall volume reduction could be caused by decreased compliance of the lungs, for example, owing to rejection of the graft, or by chest wall restriction and/or respiratory muscle weakness. If low lung compliance were the cause, we would have expected esophageal pressures at TLC to be more highly negative than normal, as, for example, in pulmonary fibrosis. However, in 5 of our subjects, esophageal pressures at TLC were less negative than normal, suggesting that postoperative chest wall volume restriction in our subjects resulted from weakness of inspiratory muscles. It is possible that inspiratory muscles, especially the diaphragm, are remodeled after transplantation, increasing optimal length by addition of sarcomeres in series within muscle fibers, a reversal of the length adaptation that occurs with chronic hyperinflation. ${ }^{21,22}$ It remains to be determined to what extent immunosuppressive medications, persistent effects of surgical trauma, or other factors may cause inspiratory muscle dysfunction contributing to chest wall volume reduction after transplantation. Importantly, although chest wall volume reduction after lung transplantation restricts the volume of 
the graft and impairs ventilatory function of the lungs, there is no clear evidence in our data that it contributes to mortality or serious morbidity. ${ }^{3-7}$

We thank Dr Marilyn Moy for help in the patient studies, Dr Carl O'Donnell for suggestions for the manuscript, and Emil Millet for creating the software used to analyze thoracic CT scans.

\section{References}

1. Stevens PM, Johnson PC, Bell RL, Beall AC Jr, Jenkins DE. Regional ventilation and perfusion after lung transplantation in patients with emphysema. N Engl J Med. 1970;282:245-9.

2. Wildevuur CR, Benfield JR. A review of 23 human lung transplantations by 20 surgeons. Ann Thorac Surg. 1970;9:489-515.

3. Marinelli WA, Hertz MI, Shumway SJ, Fox JM, Henke CA, Harmon $\mathrm{KR}$, et al. Single lung transplantation for severe emphysema. J Heart Lung Transplant. 1992;11(3 Pt 1):577-82; discussion 82-3.

4. Low DE, Trulock EP, Kaiser LR, Pasque MK, Dresler C, Ettinger N, et al. Morbidity, mortality, and early results of single versus bilateral lung transplantation for emphysema. J Thorac Cardiovasc Surg. 1992; 103:1119-26.

5. Gibbons WJ, Levine SM, Bryan CL, Segarra J, Calhoon JH, Trinkle $\mathrm{JK}$, et al. Cardiopulmonary exercise responses after single lung transplantation for severe obstructive lung disease. Chest. 1991;100:106-11.

6. Mal H, Andreassian B, Pamela F, Duchatelle JP, Rondeau E, Dubois $\mathrm{F}$, et al. Unilateral lung transplantation in end-stage pulmonary emphysema. Am Rev Respir Dis. 1989;140:797-802.

7. Mal H, Sleiman C, Jebrak G, Messian O, Dubois F, Darne C, et al. Functional results of single-lung transplantation for chronic obstructive lung disease. Am J Respir Crit Care Med. 1994;149:1476-81.

8. Brunsting LA, Lupinetti FM, Cascade PN, Becker FS, Daly BD, Martinez FJ, et al. Pulmonary function in single lung transplantation for chronic obstructive pulmonary disease. J Thorac Cardiovasc Surg. 1994;107:1337-44; discussion 44-5.

9. Cheriyan AF, Garrity ER Jr, Pifarré R, Fahey PJ, Walsh JM. Reduced transplant lung volumes after single lung transplantation for chronic obstructive pulmonary disease. Am J Respir Crit Care Med. 1995; 151(3 Pt 1):851-3.
10. Estenne M, Cassart M, Poncelet P, Gevenois PA. Volume of graft and native lung after single-lung transplantation for emphysema. Am J Respir Crit Care Med. 1999;159:641-5.

11. Ingenito EP, Evans RB, Loring SH, Kaczka DW, Rodenhouse JD, Body SC, et al. Relation between preoperative inspiratory lung resistance and the outcome of lung-volume-reduction surgery for emphysema. N Engl J Med. 1998;338:1181-5.

12. Lloyd KS, Barnard P, Holland VA, Noon GP, Lawrence EC. Pulmonary function after heart-lung transplantation using larger donor organs. Am Rev Respir Dis. 1990;142:1026-9.

13. Theodore J, Jamieson SW, Burke CM, Reitz BA, Stinson EB, Van Kessel A, et al. Physiologic aspects of human heart-lung transplantation. Pulmonary function status of the post-transplanted lung. Chest. 1984;86:349-57.

14. Glanville AR, Theodore J, Harvey J, Robin ED. Elastic behavior of the transplanted lung. Exponential analysis of static pressure-volume relationships. Am Rev Respir Dis. 1988;137:308-12.

15. Pinet $C$, Estenne M. Effect of preoperative hyperinflation on static lung volumes after lung transplantation. Eur Respir J. 2000;16:482-5.

16. Guignon I, Cassart M, Gevenois PA, Knoop C, Antoine M, Yernault JC, et al. Persistent hyperinflation after heart-lung transplantation for cystic fibrosis. Am J Respir Crit Care Med. 1995;151(2 Pt 1):534-40.

17. Lin KC, Dizner-Golab A, Thurer RL, Loring SH. Mediastinal and chest wall limitations to asymmetry of lung inflation. $J$ Appl Physiol. 2004:96:999-1004.

18. De Groote A, Van Muylem A, Scillia P, Cheron G, Verleden G, Paiva $\mathrm{M}$, et al. Ventilation asymmetry after transplantation for emphysema: role of chest wall and mediastinum. Am J Respir Crit Care Med. 2004:170:1233-8.

19. Loring SH, Leith DE, Connolly MJ, Ingenito EP, Mentzer SJ, Reilly JJ Jr. Model of functional restriction in chronic obstructive pulmonary disease, transplantation, and lung reduction surgery. Am J Respir Crit Care Med. 1999;160:821-8.

20. De Troyer A, Leduc D. Effects of single-lung inflation on inspiratory muscle function in dogs. J Physiol (Lond). Published July 13, 2006 as 10.1113/jphysiol.2006.112797.

21. Sharp JT, van Lith P, Nuchprayoon CV, Briney R, Johnson FN. The thorax in chronic obstructive lung disease. Am J Med. 1968;44:3946.

22. Similowski T, Yan S, Gauthier AP, Macklem PT, Bellemare F. Contractile properties of the human diaphragm during chronic hyperinflation. N Engl J Med. 1991;325:917-23. 